\title{
Ischaemic optic neuropathy with painful ophthalmoplegia in diabetes mellitus
}

\author{
DOUGLAS A. JABS, ' NEIL R. MILLER, ${ }^{1}$ AND W. RICHARD GREEN ${ }^{2}$ \\ From the ${ }^{1}$ Neuro-ophthalmology Unit and the ${ }^{2}$ Eye Pathology Laboratory, \\ the Wilmer Ophthalmologic Institute, and the ${ }^{2}$ Department of Pathology, \\ the Johns Hopkins Medical Institutions, Baltimore, Maryland, USA
}

SUMmaRY Two patients with mild, adult-onset diabetes mellitus developed a painful ophthalmoplegia and ipsilateral optic neuropathy that was relatively unresponsive to steroids. Histopathological study of the optic nerve of one patient revealed an extensive ischaemic infarct. There was ultimate recovery from the cranial nerve palsies in both patients and the optic neuropathy in one patient. Ischaemic polyneuropathy involving the cranial nerves may cause an orbital apex/ cavernous sinus syndrome in patients with diabetes mellitus.

Painful ophthalmoplegia associated with ipsilateral optic neuropathy is typically caused by processes involving the region of the orbital apex and cavernous sinus. The primary causes include: (1) tumours, especially metastatic carcinoma, nasopharyngeal carcinoma, and lymphoproliferative disorders; (2) vascular lesions, such as aneurysms and carotidcavernous sinus fistuli; and (3) inflammatory lesions, such as the Tolosa Hunt syndrome. We have recently seen 2 patients, both adult-onset diabetics, who developed the simultaneous onset of unilateral painful ophthalmoplegia and severe ipsilateral retrobulbar optic neuropathy relatively unresponsive to corticosteroid therapy that resolved spontaneously over 2 to 3 months. In one patient histopathological examination revealed that the cause of the optic neuropathy was an ischaemic infarct in the intracanalicular optic nerve.

\section{Case reports}

CASE 1

A 48-year-old woman presented with a 2-week history of severe right-sided retro-orbital pain and headache.

Presented in part before the Thirty-ninth Clinical Meeting of the Residents Association of the Wilmer Ophthalmologic Institute, Baltimore, 3 May 1980.

Correspondence to Dr Neil R. Miller, the Wilmer Ophthalmologic Institute, Room B-16, Johns Hopkins Hospital, 601 N Broadway, Baltimore, Maryland 21205, USA
Her past medical history was significant for systemic hypertension, episodic congestive heart failure, and mild diabetes mellitus. Three days previously she had noted blurred vision in the right eye.

On examination the patient had a visual acuity of $6 / 12(20 / 40)$ in the right eye and 6/6 (20/20) in the left eye. She could identify only 2 of 9 Hardy-Rand-Rittler (HRR) colour plates with the right eye but 9 out of 9 plates with the left eye. There was a right afferent pupillary defect. Examination of the right visual field disclosed a large peripheral nasal defect and a central scotoma. The left visual field was full. Both fundi were normal. The patient was considered to have an optic neuritis. A skull $x$-ray was normal. An erythrocyte sedimentation rate was $20 \mathrm{~mm} / \mathrm{h}$, and the fasting blood glucose was $158 \mathrm{mg} / \mathrm{dl}(8 \cdot 8 \mathrm{mmol} / \mathrm{l})$.

Over the next 3 days the patient's visual acuity rapidly deteriorated, and she was re-examined. The right eye now had no light perception. The left eye had 6/6 (20/20) visual acuity. there was a slight right ptosis, a 15 dioptre right hypertropia, and decreased abduction of the right eye. There was no proptosis. The fundi remained normal. The patient was admitted to the Johns Hopkins Hospital for further evaluation.

On admission a neurological examination was normal. A complete blood count and differential examination were normal. A serological test for syphilis and a test for antinuclear antibodies and LE preparation were all negative. Fasting blood glucose was mildly elevated. Plain $x$-rays of the skull, paranasal sinuses, and orbits were normal. Orbital 

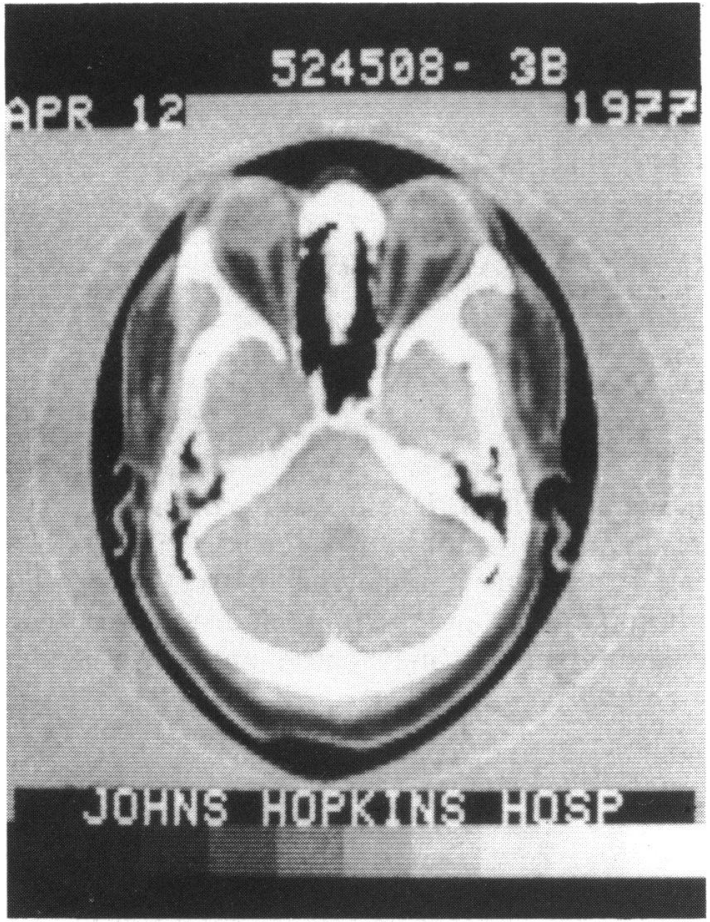

Fig. 1 Case 1. Unenhanced computerised tomographic scan showing normal orbital and intracranial contents. There is no evidence of a mass lesion.

polytomography, radionuclide scan, and gallium scan were normal. A computerised tomographic scan showed no evidence of an orbital or intracranial mass (Fig. 1). A lumbar puncture showed cerebrospinal

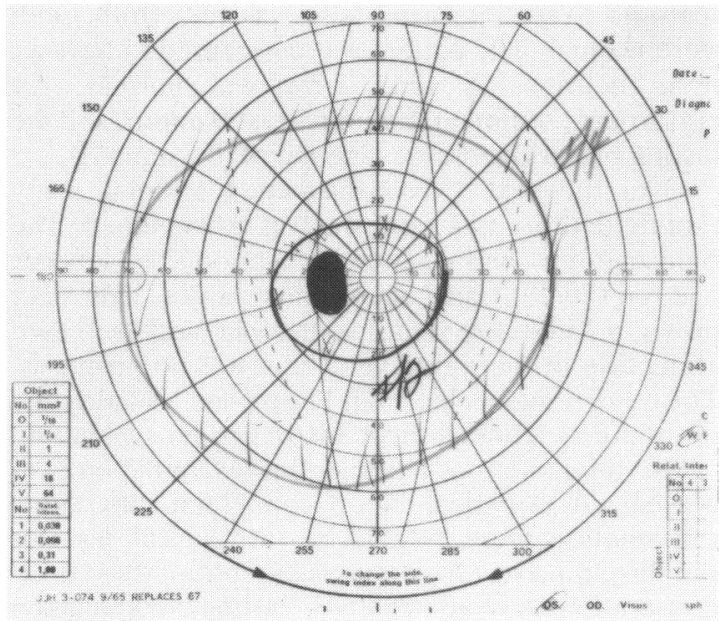

fluid (CSF) under normal pressure. There were no cells in the CSF but the protein content was raised to $115 \mathrm{mg} / \mathrm{dl}(1 \cdot 15 \mathrm{~g} / \mathrm{l})$. The CSF VDRL was negative.

One day after admission, the patient was begun on $80 \mathrm{mg}$ of prednisone per day. Over the next 5 days visual acuity in the right eye improved to $6 / 120$ $(20 / 400)$, and within 2 weeks visual acuity had improved to $6 / 60(20 / 200)$ with an inferior nasal quadrantic field defect. The patient's pain continued, however, as did her ophthalmoplegia. She refused an arteriogram and signed out of the hospital against medical advice. Two days later she was readmitted with a blood glucose of $600 \mathrm{mg} / \mathrm{dl}(33 \mathrm{mmol} / \mathrm{l})$. Examination showed her to be otherwise unchanged. She again refused further evaluation. For this reason prednisone was rapidly tapered and discontinued. She was discharged from the hospital and lost to follow-up.

Two and one-half months later the patient returned to the Wilmer Ophthalmological Institute for reevaluation. Visual acuity had improved to $6 / 6(20 / 20)$ in the right eye. There was no ptosis, extraocular movements were full, and the patient was orthophoric. The right visual field continued to show an inferior nasal quadrantic field defect (Fig. 2).

CASE 2

A 57-year-old man complained of a left frontaltemporal headache of 2 months' duration. Examination at a local hospital revealed a fasting blood glucose of $260 \mathrm{mg} / \mathrm{dl}(14.4 \mathrm{mmol} / \mathrm{l})$. The patient was admitted to the hospital for evaluation of his diabetes mellitus. However, on the second hospital day he experienced sudden onset of oblique diplopia as well as blurred vision in the left eye. Over the next 7 days the visual

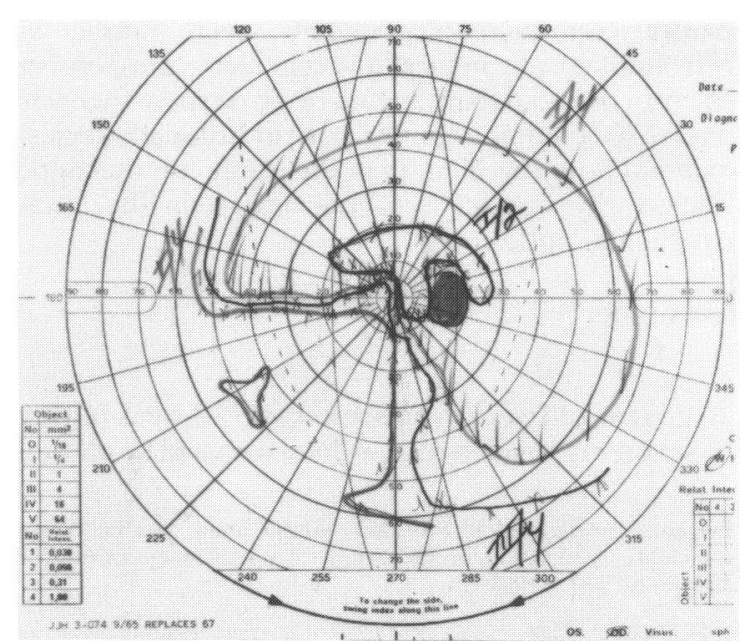

Fig. 2 Case 1 (left and right). Visual fields as performed on a Goldmann perimeter. There is an inferior nasal quadrantic defect in the field of vision of the right eye. 

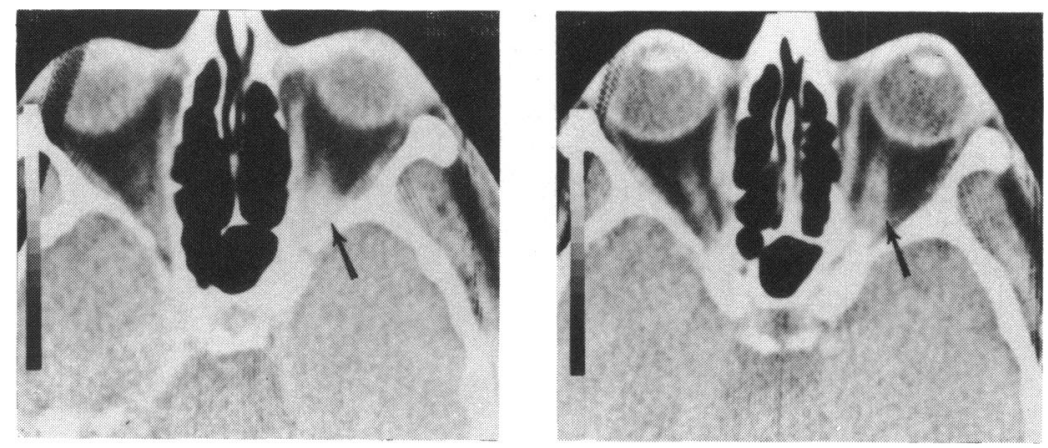

Fig. 3 Case 2. Unenhanced computerised tomographic scan. A. There is an apparent, ill-defined mass in the posterior left orbit (arrow). B. At a different level there is apparent thickening of the posterior left optic nerve (arrow).

acuity rapidly diminished in the left eye. A computerised tomographic scan showed an apparent diffuse mass in the posterior aspect of the left orbit, and the patient was transferred to the Wilmer Ophthalmological Institute for evaluation.

On initial examination visual acuity in the right eye was $6 / 5(20 / 15)$. There was no perception of light in the left eye. The visual field of the right eye was full. The pupils were equal. The right pupil was normally reactive to light and near vision. The left pupil was unreactive to direct light but reacted consensually. There was a moderate left ptosis. There was complete limitation of adduction and depression, and moderate limitation of elevation and abduction in the left eye. The left eye was proptosed $2.5 \mathrm{~mm}$. Forced ductions were performed and showed no mechanical limitation of motion of the left eye. Ophthalmoscopy revealed normal fundi. A computerised tomographic scan was repeated and again showed a diffuse density in the posterior left orbit. In addition, the optic nerve appeared to be slightly thickened (Fig. 3). Stool examination, sigmoidoscopy, an otolaryngological examination, and a chest $\boldsymbol{x}$-ray were all normal. Sputum cytology showed mild squamous atypia.

The patient was treated with prednisone at a dosage of $80 \mathrm{mg}$ per day for 4 days. Although his pain diminished, neither his visual acuity nor his extraocular movements improved. For this reason, and because of the possibility of an underlying neoplastic process he underwent neurosurgical exploration approximately 3 weeks after admission. At the time of surgery the optic canal and the posterior orbit were unroofed. No abnormality was found in the orbit. The intracanalicular optic nerve was erythematous, swollen, and densely adherent to the medial and lateral undersurface of the optic canal. A biopsy of the optic nerve was performed, and frozen sections were read as consistent with an optic nerve glioma. Because the patient was completely blind in the left eye and had been so for approximately one month the entire length of the intracanalicular optic nerve was removed for pathological examination. Over the next 3 months the patient's pain disappeared, and he had full recovery of extraocular motion and disappearance of ptosis.

Microscopic examination of permanent sections of the optic nerve disclosed ischaemic necrosis involving an entire portion of the distal area of the excised segment (Fig. 4). There appeared to be a discrete delineation between the infarcted and normal areas (Fig. 4) but closer examination revealed several transitional zones. The infarcted area was characterised by loss of most of the cell, myelin and axons (Fig. 5). Some peripheral areas of the infarcted zone were infiltrated by numerous histiocytes with markedly distended cytoplasm containing a finely granular material (Fig. 6). Adjacent to these regions were zones of distended astrocytes and microglia (Fig. 7), followed by zones of prominent vascular congestion and minor glial hypercellularity. The blood vessels in

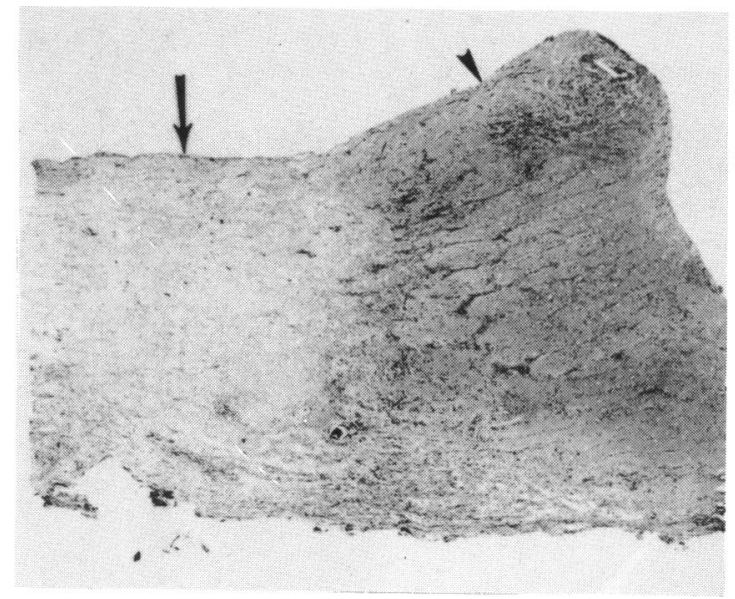

Fig. 4 Optic nerve showing abrupt transition between infarcted area (arrow) and relatively normal zone (arrowhead). (Haematoxylin and eosin, $\times 14$ ). 


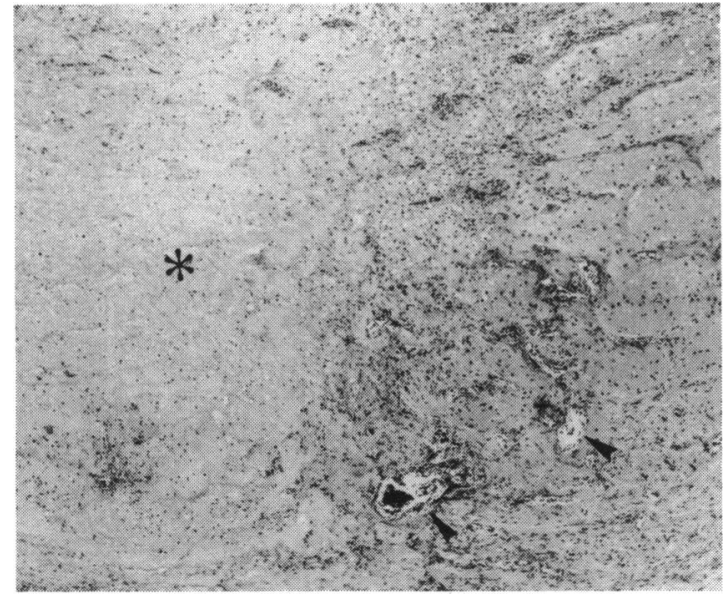

Fig. 5 Higher power showing transitional zone between infarcted area (asterisk) and normal area. There is vascular dilatation (arrowhead) and round cell infiltration.

(Haematoxylin and eosin, $\times 33$ ).

this zone appeared distended and some had lymphocytic infiltration in their walls and surrounding tissue (Fig. 5). In one area a large pial vessel extending into the nerve also had a prominent lymphocytic infiltration (Fig. 8). Careful examination disclosed no occluded vessels. Multiple specimens taken from the posterior orbit showed normal fat and connective tissue with no evidence of either tumour or inflammation.

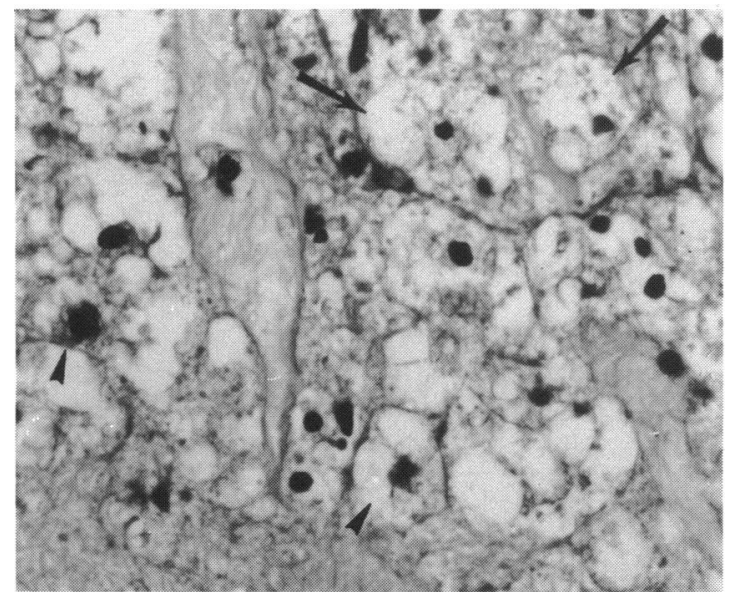

Fig. 7 Transitional zone showing distended astrocytes (arrowheads) and microglia (arrows). (Haematoxylin and eosin, $\times 523$ ).

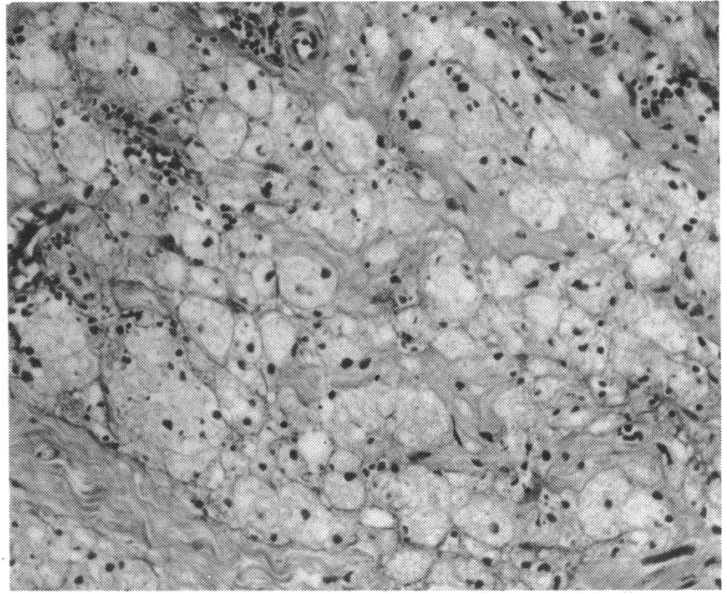

Fig. 6 Peripheral zone of infarcted area with an infiltration of greatly distended histiocytes. (Haematoxylin and eosin, $\times 183)$.

\section{Discussion}

These 2 patients present similar clinical pictures. In both cases the patient was a middle-aged adult with mild diabetes mellitus. Visual acuity was lost over a period of one week and reduced to no light perception there were multiple, unilateral, painful cranial nerve palsies involving both the third and sixth nerves; the optic disc appeared normal on initial examination;

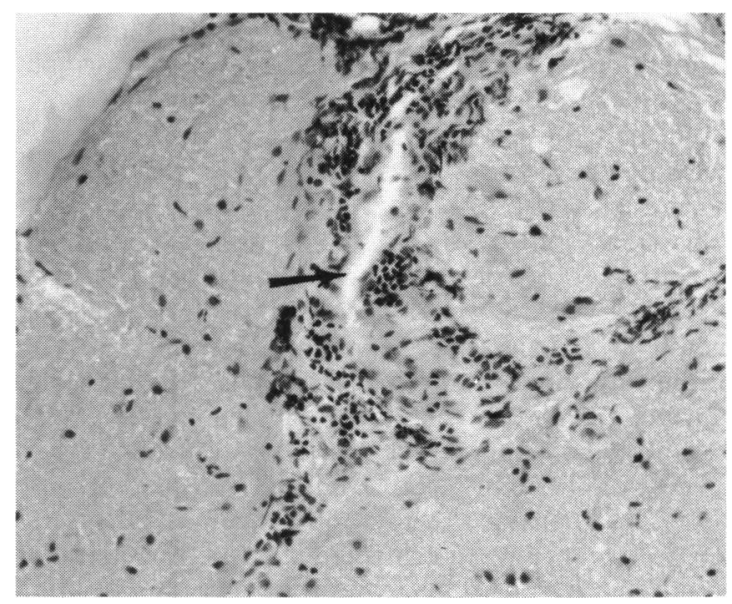

Fig. 8 Perivascular lymphocytic infiltration of pial vessel (arrow) extending into noninfarcted area. (Haematoxylin and eosin, $\times 150)$. 
and there was complete recovery of the cranial nerve palsies. In each case corticosteroids were used with improvement but not resolution of pain. The first patient had some improvement in visual acuity initially after steroids, but this may have been the natural evolution of the disease rather than a response to the steroids. Furthermore, over the next 3 weeks there was little further improvement in her visual acuity even while on prednisone. After discontinuation of the steroids there was subsequent recovery to normal visual acuity and to normal cranial nerve function. In the second patient there was no improvement in either visual acuity or ophthalmoplegia with corticosteroids.

The histopathology of the optic nerve in the second patient is that of an infarct of greater than 14 days' duration; and the clinical presentation, pathological findings, and subsequent recovery of the third and sixth nerves are consistent with a diabetic neuropathy.

There are 3 previous pathological reports on diabetic cranial nerve palsies, and the pathology in each case is that of an ischaemic process. In the case reported by Dreyfus et al. ${ }^{1}$ the ocular motor nerve was markedly swollen to almost twice its normal size. Microscopic examination revealed incomplete necrosis of the involved tissue, interstitial oedema, and an infiltrate of macrophages. In the case reported by Asbury et al. ${ }^{2}$ microscopic examination of the recently involved left third nerve revealed a focal lesion in the intracavernous portion of the nerve. This lesion was a noninflammatory, primarily demyelinating process with a sharp proximal border but an indistinct distal border. Ischaemia was also felt to be the cause of this lesion. Interestingly, no pathological abnormalities were found in the opposite ocular motor nerve, which had been transiently paralysed several years previously. Weber $e t$ al. $^{3}$ demonstrated a fusiform swelling in the subarachnoid portion of the third nerve with an abrupt transition between normal and abnormal tissue. Microscopically there was a distinct decrease in stainable myelin and a slight decrease in the number of axons.

The histopathology of the optic nerve in our case 2 is that of an infarction with changes similar to those seen in the oculomotor nerve examined by Dreyfus $e t$ al. ${ }^{1}$ We assume that both the left oculomotor and abducens nerves in our patient were involved in a similar, although not so severe, process.

There is no histopathological evidence to suggest that the posterior orbital contents, other than the optic, oculomotor, and abducens nerves, were involved in the ischaemic process. The increased density seen on computerised tomography and mistakenly assumed to represent a neoplastic or inflammatory process may have simply been a tissue response to the ischaemic optic and ocular motor neuropathy. It is conceivable that our case 1 had a less severe lesion, perhaps similar to the demyelinating process reported by Asbury et al., ${ }^{2}$ and thus had a normal computerised tomographic scan.

Although diabetes mellitus is a well known cause of isolated, often painful, cranial nerve palsies, it is less well appreciated that multiple simultaneous cranial nerve palsies also occur in diabetics.

In 1929 Collier $^{4}$ commented on having seen diabetic patients with simultaneous paralyses of the third and fourth cranial nerves on one side and discussed in detail a 64-year-old woman with glycosuria for years who initially developed a palsy of the right third and fourth nerves. Her ocular motility completely recovered within 4 months. However, 6 months later she developed an acute, left 'retrobulbar neuritis' associated with complete paralyses of the left third and fourth nerves. There was recovery of ocular motility but not visual acuity. This case appears to be similar to our case 2 . In his report Collier also quotes. Dieulafoy as having seen several cases of multiple cranial nerve pareses in patients with diabetes mellitus, but it is not clear whether these pareses were simultaneous or not.

In 1935 Jordan $^{5}$ reported a 64-year-old man with the onset over 1 to 3 days of paralysis of both superior recti as well as the left lateral rectus. Walsh and $\mathrm{Hoyt}^{6}$ describe a 57-year-old woman with diabetes mellitus who presented with the simultaneous onset of a third and sixth nerve paralysis of the left eye. They also describe a diabetic patient with total unilateral external ophthalmoplegia and sensory loss in the distribution of the trigeminal nerve. Ross ${ }^{7}$ has reported 3 patients with diabetes mellitus and recurrent cranial nerve palsies. His first case presented with palsies of the third, fourth, and sixth cranial nerves on the right and subsequently developed palsies of the third, fourth, and sixth cranial nerves on the left side. Eventually there was full recovery on each side. Ross's third patient had many episodes of complete as well as partial right and left third and sixth nerve paralyses. Larsen and Auchincloss ${ }^{8}$ have reported 3 patients with multiple, bilateral, symmetrical cranial nerve palsies, involving various combinations of cranial nerves $3,5,6,7,9,10$, and 11 .

Causes of anterior cavernous sinus/orbital apex syndromes include aneurysms, tumours, and inflammatory lesions. No evidence for either an aneurysm or a neoplasm was found in either of our cases, and in both the condition resolved. The Tolosa Hunt syndrome deserves special mention. In 1954 Tolosa9 and in 1961 Hunt et al. ${ }^{10}$ described a syndrome characterised by recurrent unilateral painful ophthalmoplegia. Pathological examination ${ }^{9-12}$ of such cases has revealed granulomatous inflammation in the anterior cavernous sinus. The process is exquisitely sensitive to 
steroids, prompting Smith and Taxdal ${ }^{13}$ to propose a 48-hour trial of high-dose steroids as both a diagnostic test and a therapeutic trial. In both of our patients there was some decrease in pain after corticosteroid therapy was instituted but little other effect in case 1 and none in case 2 . A primary inflammatory orbital lesion (pseudotumour) thus does not appear to have been present in either case. In case 1 there was no radiographic evidence of an orbital inflammation. In case 2, although there was an increased density by computerised tomography, multiple biopsies of posterior orbital tissue failed to demonstrate any evidence of inflammation. In any event the optic nerve pathology in case 2 is ischaemic, not inflammatory. Although there were some histiocytes within the optic nerve, these cells are secondary to ischaemia rather than to a primary inflammatory process.

It would thus appear likely that both of our patients developed unilateral painful ophthalmoplegia and optic neuropathy as a result of the microangiopathy associated with diabetes mellitus. While we would caution that this 'orbital apex/cavernous sinus' syndrome is rare, it should be kept in mind in patients with diabetes mellitus in whom other causes have been ruled out and who do not respond dramatically to corticosteroid therapy.

\section{References}

1 Dreyfus PM, Hakim S, Adams RD. Diabetic ophthalmoplegia: report of case, with postmortem study and comments on vascular supply of human oculomotor nerve. Arch Neurol (Psychiatry) 1957; $77: 337-49$.

2 Asbury AK, Aldredge H, Hershberg R, Fisher CM. Oculomotor palsy in diabetes mellitus: a clinicopathologic study. Brain 1970; 93: 555-66.

3 Weber RB. Daroff RB, Mackey EA. Pathology of oculomotor nerve palsy in diabetics. Neurology 1970; 20: 835-8.

4 Collier J. Paralysis of the oculomotor nerve-trunks in diabetes. Proc $R$ Soc Med 1930; 23: 627-30.

5 Jordan WR. Neuritic manifestations in diabetes mellitus. Arch Intern Med 1936; 57: 307-58.

6 Walsh FB. Hoyt WF. Clinical Neuro-Ophthalmology. 3rd ed. Baltimore: Williams and Wilkins, 1969: 1109-20.

7 Ross AT. Recurrent cranial nerve palsies in diabetes mellitus Neurology 1962; 12: 180-5.

8 Larson DL. Auchincloss JH. Multiple symmetric bilateral cranial nerve palsies in patients with unregulated diabetes mellitus: report of three cases. Arch Intern Med 1950; 85: 265-71.

9 Tolosa E. Periarteritic lesions of the carotid siphon with the clinical features of a carotid infraclinoidal aneurysm. $J$ Neurol Neurosurg Psychiatry 1954 19: 300-2.

10 Hunt W, Meagher JN, Le Fever HE. Zeman W. Painful ophthalmoplegia: its relation to indolent inflammation of the cavernous sinus. Neurology 1961; 11: 56-62

11 Lahke J. Superior orbital fissure syndrome. Arch Neurol 1962: 7: 289-300.

12 Hunt WE. Tolosa Hunt syndrome: one cause of painful ophthalmoplegia. J Neurosurg 1976; 44: 544-9.

i3 Smith JL. Taxdal DR. Painful ophthalmoplegia. The Tolosa Hunt syndrome. Am J Ophthalmol 1966; 61: 1466-72. 